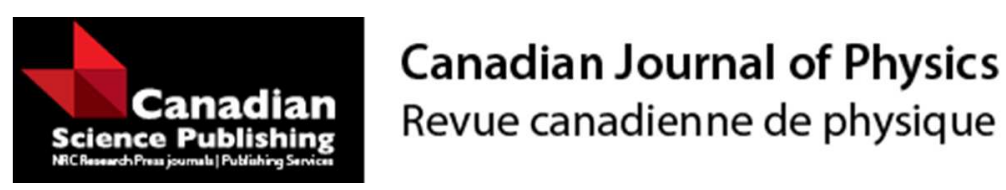

\title{
Path integral treatment of the deformed Schioberg-type potential for some diatomic molecules
}

\begin{tabular}{|r|l|}
\hline Journal: & Canadian Journal of Physics \\
\hline Manuscript ID & cjp-2016-0397.R2 \\
\hline Manuscript Type: & Article \\
\hline Date Submitted by the Author: & $28-$ Sep-2016 \\
\hline Complete List of Authors: & $\begin{array}{l}\text { Amrouche, Assia; Universite Dr Yahia Fares de Medea } \\
\text { Diaf, Ahmed; University of Khemis Miliana, } \\
\text { Hachama, Mohamed; University of Khemis Miliana, Mathematics and } \\
\text { Informatics }\end{array}$ \\
\hline Keyword: & $\begin{array}{l}\text { Path integral, I-states, deformed Schioberg-type potential, Duru-Kleinert } \\
\text { space-time transformation, Diatomic molecules }\end{array}$ \\
\hline
\end{tabular}




\title{
Path integral treatment of the deformed Schiöberg- type potential for some diatomic molecules
}

\author{
Assia Amrouche, Ahmed Diaf, and Mohammed Hachama
}

\begin{abstract}
The bound state solution of the Feynman propagator with the deformed generalized Schiöberg potential is determined using an approximation of the centrifugal term. The energy eigenvalue expression is computed using DuruKleinert space-time transformation for both positive and negative deformation parameters of diatomic molecules. Besides, the rotation-vibration energy eigenvalues are numerically calculated for some diatomic molecules and compared with those given in the literature. The obtained results are in agreement with those given by state-of-the-art approximate and numerical methods.
\end{abstract}

Key words: Path integral, deformed Schiöberg-type potential, $l$-states.

\section{Introduction}

The study of analytical solutions for empirical potential functions of diatomic molecules has been the object of many investigations for several reasons. For instance, in chemical physics [1], an accurate potential energy curve is needed in the evaluation of spectroscopic constants and reactive scattering theoretical studies. In addition, accurate vibrational energies and wave functions are required in studies such as the vibrational excitation of electron-molecule collision which involves molecular vibrational excitation process.

Several potential functions has been proposed in the literature. A first simple empirical analytical potential function was proposed by Morse [2] and has been employed in a wide variety of problems in chemical physics such as in molecular spectroscopy [3] and in molecular dynamics simulation [4]. Another famous hyperbolic type potential is the Schiöberg potential [5] which includes the Morse, Kratzer and Coulomb potentials as special cases. More general potential functions have been constructed by Eğrifes et al. [10, 11, 12], based on the deformed hyperbolic functions [9], and which are identical to the Tietz potential model for diatomic molecules [13, 14]. These q-deformed potentials have been widely used in different applications such as the quantum statistical theory [15], the conformal field theory [16, 17], the nuclear structures [18], and the chemical physics [19]. For the above potentials, the Schrödinger's equation has been exactly solved for the $s$ states. For $\ell \neq 0$ states, several approximate techniques have been proposed. We can cite the Nikiforov-Uvarov method [6], the function analysis [7], the asymptotic iteration [8], and the Feynman path integrals $[24,25,26]$. In recent papers, some q-deformed empirical potentials have been addressed, such as Woods-Saxon [20], the four-parametric deformed Schiöberg type $[21,22]$ and the q-deformed hyperbolic Poschl-Teller potential [23].

Recently, Mustafa introduced a four-parametric deformed

A. Amrouche. Faculty of Science and Technology, Médéa University, 26000, Algeria.

A. Diaf and M. Hachama. Laboratory of Energy and Intelligent Systems, University of Khemis Miliana, 44225, Khemis Miliana, Algeria
Schiöberg-type potential for diatomic molecules [21]. He computed the energy spectrum of the $\ell$-states using the supersymmetric quantization in the Schrödinger framework. In this paper, we use the Feymann path integral formalism to solve this potential. In Section 2, we discuss the deformed Schiöbergtype potential and show some properties. In Section 3, the Feynman propagator is investigated using the Duru-Kleinert method to obtain the energy eigenvalues. In Section 4, numerical results for some diatomic molecules are given and compared with previous ones [21,27]. Finally, some concluding remarks are given in Section 5.

\section{The Schiöberg-type potential}

Based on the original expression of the Schiöberg potential [5], Mustafa [21] has proposed the four-parametric deformed Schiöberg-type potential:

$U(r)=A\left[B+\tanh _{q}(\alpha r)\right]^{2}$,

where the $q$-deformation of the usual hyperbolic functions are defined by the following expressions:

$$
\begin{aligned}
\tanh _{q}(\alpha r) & =\frac{\sinh _{q}(\alpha r)}{\cosh _{q}(\alpha r)}, \\
\sinh _{q}(\alpha r) & =\frac{e^{\alpha r}-q e^{-\alpha r}}{2}, \\
\cosh _{q}(\alpha r) & =\frac{e^{\alpha r}+q e^{-\alpha r}}{2},
\end{aligned}
$$

On the other hand, empirical potential functions for diatomic molecules satisfy the following Varchni's conditions [28]:

$$
\begin{aligned}
\left.\frac{d U(r)}{d r}\right|_{r=r_{e}} & =0, \\
U(\infty)-U\left(r_{e}\right) & =D_{e}, \\
\left.\frac{d^{2} U(r)}{d r^{2}}\right|_{r=r_{e}} & =K_{e} \equiv(2 \pi c)^{2} \mu \omega_{e}^{2},
\end{aligned}
$$

where $D_{e}$ is the dissociation energy, $r_{e}$ is the equilibrium bond length, $c$ is the speed of light, $\mu$ is the reduced mass, and $\omega_{e}$ is the equilibrium harmonic oscillator vibrational frequency. 
Using Varshni's conditions (3) and (4), we can rewrite the potential (1) in the following form:

$U(r)=D_{e}\left[1-\frac{e^{2 \alpha r_{e}}+q}{e^{2 \alpha r}+q}\right]^{2}$.

This improved Tietz potential has also been obtained for diatomic molecules in [29]. The analytical solutions of the Schrödinger equation with this potential have been investigated [30,31].

Furthermore, applying Varshni's condition (5) gives:

$$
\begin{aligned}
q & =-\left(1-\frac{2 \alpha}{\beta}\right) e^{2 \alpha r_{e}} \\
& =-\eta e^{2 \alpha r_{e}}
\end{aligned}
$$

with $\beta=\sqrt{\frac{K_{e}}{2 D_{e}}}$ and $\eta=\left(1-\frac{2 \alpha}{\beta}\right)$.

On the other hand, the Tietz-Hua oscillator potential [27] is given by:

$U(r)=D_{e}\left[\frac{1-e^{-b_{h}\left(r-r_{e}\right)}}{1-c_{h} e^{-b_{h}\left(r-r_{e}\right)}}\right]^{2}$

where $c_{h}$ is an optimization parameter and $b_{h}=\beta\left(1-c_{h}\right)$. This potential is equivalent to the deformed Schiöberg potential (6) where $\eta=c_{h}$ and $2 \alpha=b_{h}$ as shown in [21]. In next Section, we will take advantage of this equivalence when solving the potential (6) with the Feynman path integral technique by using the spectroscopic parameters of the diatomic molecules given in Table 1.

\section{Solving the Schiöberg-type potential with the path integral method}

The basic idea for solving the studied potential with the path integral method is to find an appropriate space-time transformation to reformulate the initial problem in terms of a wellknown and already solved problem. Actually, many unknown path integrals have been solved by using their relation to known ones. This method has been discussed in detail in other papers (See references [24, 25, 26]). We present here a brief summary of the essential steps involved.

Let's consider a spherically symmetric effective potential $U_{\text {eff }}$ defined by the following:

$U_{e f f}\left(r_{j}, \ell\right)=U\left(r_{j}\right)+\frac{\ell(\ell+1) \hbar^{2}}{2 \mu r^{2}}$.

The propagator for a particle of mass $\mu$ in the potential $U_{\text {eff }}$ can be developed into a sum of partial waves of the form:

$$
\begin{aligned}
& k\left(r^{\prime \prime}, t^{\prime \prime} ; r^{\prime}, t^{\prime}\right)= \\
& \frac{1}{4 \pi r^{\prime \prime} r^{\prime}} \sum_{\ell=0}^{\infty}(2 \ell+1) K_{\ell}\left(r^{\prime \prime}, t^{\prime \prime} ; r^{\prime}, t^{\prime}\right) P_{\ell}(\cos \theta),
\end{aligned}
$$

where $P_{\ell}(\cos \theta)$ is the Legendre polynomial and the partial propagator $K_{\ell}$ is defined by

$$
\begin{aligned}
& K_{\ell}\left(r^{\prime \prime}, t^{\prime \prime} ; r^{\prime}, t^{\prime}\right)= \\
& \lim _{N \rightarrow \infty} \int \prod_{j=1}^{N} \exp \left[\frac{i}{\hbar} S_{j}\right] \prod_{j=1}^{N}\left[\frac{\mu}{2 \pi i \hbar \varepsilon}\right]^{\frac{1}{2}} \prod_{j=1}^{N-1} d r_{j},
\end{aligned}
$$

with $S_{j}=\frac{\mu}{2 \varepsilon}\left(\Delta r_{j}\right)^{2}-\varepsilon U_{e f f}\left(r_{j}, \ell\right), \Delta r_{j}=r_{j}-r_{j-1}, \varepsilon=$ $t_{j}-t_{j-1}, t^{2 \varepsilon}=t_{0}$ and $t^{\prime \prime}=t_{N}$.

This path integral (12) is not solvable for nonzero angular momentum states $(\ell \neq 0)$. To overcome this difficulty, we use an approximation for $\frac{1}{r^{2}}$ centrifugal, given by

$\frac{r_{e}^{2}}{r^{2}}=C_{0}+\frac{C_{1}}{\left(e^{2 \alpha r}+q\right)}+\frac{C_{2}}{\left(e^{2 \alpha r}+q\right)^{2}}$.

The constants $C_{i}$ can be obtained using a factorization recipe as done in $[32,33]$ :

$$
\begin{aligned}
& C_{1}=1-\left(\frac{1-\eta}{u}\right)^{2}\left[\frac{4 u}{1-\eta}-(3+u)\right] \\
& C_{2}=2 e^{u}(1-\eta)\left[3\left(\frac{1-\eta}{u}\right)-(3+u)\left(\frac{1-\eta}{u}\right)^{2}\right] \\
& C_{2}=\frac{e^{2 u}}{u^{2}}(1-\eta)^{4}\left[(3+u)-\frac{2 u}{1-\eta}\right] .
\end{aligned}
$$

From Table 1, we note that $\eta$ can take positive or negative values and so is $q=-\eta e^{2 \alpha r_{e}}$. Consequently, the effective potential given in (10) can be put in the following form:

$$
U_{e f f}\left(r_{j}, \ell\right)=\widetilde{P}_{1}+\frac{\widetilde{P}_{2}}{\left(e^{2 \alpha r_{j}}+q\right)}+\frac{\widetilde{P}_{3}}{\left(e^{2 \alpha r_{j}}+q\right)^{2}},
$$

with

$$
\begin{aligned}
\gamma & =\frac{\ell(\ell+1) \hbar^{2}}{2 \mu r_{e}^{2}}, b=2 \alpha \\
P_{1} & =D_{e}, P_{2}=-2 D_{e}\left(e^{2 \alpha r_{e}}+q\right) \\
P_{3} & =D_{e}\left(e^{2 \alpha r_{e}}+q\right)^{2} \\
\widetilde{P}_{1} & =P_{1}+\gamma C_{1}, \widetilde{P}_{2}=P_{2}+\gamma C_{2}, \widetilde{P}_{3}=P_{3}+\gamma C_{3} .
\end{aligned}
$$

Depending on the sign of $\eta$, we will consider two cases in the following. We will show that these cases correspond to the deformed Manning-Rosen and the deformed Rosen-Morse potentials. Such a discussion has also been made previously for the particular case of the standard Manning-Rosen and RosenMorse potentials in [34].

3.1. Case $\eta<0(q>0)$

The potential (15) can be written as

$U_{e f f}\left(r_{j}, \ell\right)=A \tanh _{q}\left(\alpha r_{j}\right)-\frac{B(\ell)}{\cosh _{q}^{2}\left(\alpha r_{j}\right)}+C(\ell)$, 


\begin{tabular}{cccccc}
\hline \hline Molecule & $\eta$ & $\mu / 10^{-23}(\mathrm{~g})$ & $\alpha\left(\AA^{-1}\right)$ & $r_{e}(\AA)$ & $D_{e}\left(\mathrm{~cm}^{-1}\right)$ \\
\hline$N O\left(X^{2} \Pi_{r}\right)$ & 0.013727 & 1.249 & 1.357795 & 1.151 & 53341 \\
$O_{2}\left(X^{3} \Sigma_{g}^{-}\right)$ & 0.027262 & 1.377 & 1.295515 & 1.207 & 42041 \\
$O_{2}^{+}\left(X^{2} \Pi_{g}\right)$ & -0.019445 & 1.377 & 1.434935 & 1.116 & 54688 \\
$N_{2}\left(X^{1} \Sigma_{g}^{+}\right)$ & -0.032325 & 1.171 & 1.392925 & 1.097 & 79885 \\
$H_{2}\left(X^{1} \Sigma_{g}^{+}\right)$ & 0.170066 & 0.084 & 0.80945 & 0.741 & 38318 \\
$H F\left(X^{1} \Sigma^{+}\right)$ & 0.127772 & 0.160 & 0.971035 & 0.917 & 49382 \\
\hline \hline
\end{tabular}

Table 1. Spectroscopic molecular parameters for diatomic molecules.

where

$$
A=-\frac{\widetilde{P}_{2}}{2 q}-\frac{\widetilde{P}_{3}}{2 q^{2}}, B(\ell)=\frac{\widetilde{P}_{3}}{4 q}, \quad C(\ell)=\widetilde{P}_{1}+\frac{\widetilde{P}_{2}}{2 q}+\frac{\widetilde{P}_{3}}{2 q^{2}} .
$$

Using the translation $y=r_{j}-\frac{1}{\alpha} \ln \sqrt{q}$, we can easily find the non-deformed potential:

$U_{e f f}\left(r_{j}, \ell\right)=A \tanh (\alpha y)-\frac{B^{\prime}(\ell)}{\cosh ^{2}(\alpha y)}+C(\ell)$,

where

$$
A=-\frac{\widetilde{P}_{2}}{2 q}-\frac{\widetilde{P}_{3}}{2 q^{2}}, B^{\prime}(\ell)=\frac{\widetilde{P}_{3}}{4 q^{2}}, \quad C(\ell)=\widetilde{P}_{1}+\frac{\widetilde{P}_{2}}{2 q}+\frac{\widetilde{P}_{3}}{2 q^{2}} .
$$

The effective potential given by (18) is solved in [26] by the Duru-Kleinert method, based on the nonlinear space-time transformation

$\left\{\begin{array}{rlr}y & = & f(z) \\ d t & = & {\left[f^{\prime}(z)\right]^{2} d \mathfrak{s}}\end{array}\right.$

where $(z, \mathfrak{s})$ are the new space-time coordinates and

$$
f(z)=\frac{1}{\alpha} \operatorname{arctanh}\left[2 \tanh ^{2} z-1\right] .
$$

Therefore, we can write the propagator associated to the potential (18) in terms of the modified Pöschl-Teller one which is solvable and allows us to compute the following energy spectrum [26]:

$$
E_{n, \ell}^{D S c h}=-\frac{\hbar^{2} \alpha^{2}(s-2 n-1)^{2}}{8 \mu}-\frac{2 \mu A^{2}}{\hbar^{2} \alpha^{2}(s-2 n-1)^{2}}+C(\ell),
$$

with

$s=\sqrt{1+\frac{8 \mu B^{\prime}(\ell)}{\hbar^{2} \alpha^{2}}}$.

3.2. Case $\eta>0(q<0)$

In terms of hyperbolic functions, the effective potential (15) takes the form

$$
\begin{aligned}
U_{e f f}\left(r_{j}, \ell\right) & =A \operatorname{coth}_{q}\left(\alpha r_{j}\right)-\frac{B(\ell)}{\sinh _{q}^{2}\left(\alpha r_{j}\right)}+C(\ell), \\
& =A \operatorname{coth}(\alpha y)-\frac{B^{\prime}(\ell)}{\sinh ^{2}(\alpha y)}+C(\ell),
\end{aligned}
$$

where $y=r_{j}-\frac{1}{\alpha} \ln \sqrt{-q}$. This potential is similar to the Manning-Rosen one. To solve it, we use the following transformation, as done in [24, 25]:

$y=\frac{1}{\alpha} \operatorname{arccoth}\left[2 \operatorname{coth}^{2} z-1\right]$.

This leads us to following spectrum

$E_{n, \ell}^{D S c h}=-\frac{\hbar^{2} \alpha^{2}(s+2 n+1)^{2}}{8 \mu}-\frac{2 \mu A^{2}}{\hbar^{2} \alpha^{2}(s+2 n+1)^{2}}+C(\ell)$.

Results (25) and (30) can be combined into one expression

$E_{n, \ell}^{D S c h}=-\frac{\hbar^{2} \alpha^{2}(s \pm 2 n \pm 1)^{2}}{8 \mu}-\frac{2 \mu A^{2}}{\hbar^{2} \alpha^{2}(s \pm 2 n \pm 1)^{2}}+C(\ell)$.

The positive and the negative signs $( \pm)$ in (26) correspond to negative and positive values of $q$ respectively.

\section{Numerical results and discussions}

We show on Figure 1 the variation of the four parameter potential (6). Clearly, the depth of the potential remains constant when the parameter $q$ increases from -1 to 1 (for instance).

We evaluated the accuracy of the energy levels expression (26) for different diatomic molecules: $H_{2}\left(X^{1} \Sigma_{g}^{+}\right), H F\left(X^{1} \Sigma^{+}\right)$, $N_{2}\left(X^{1} \Sigma_{g}^{+}\right), N O\left(X^{2} \Pi_{r}\right), O_{2}\left(X^{3} \Sigma_{g}^{-}\right)$and $O_{2}^{+}\left(X^{2} \Pi_{g}\right)$. We took here molecules with positive and negative values of $q$. We compared our results to those obtained with state-of-theart techniques: the numerical method [27] (reference method providing a ground truth), and the supersymetric quantization (SSQ) (with the same approximation of the barrier) [21]. Results are reported in Table 2, 3, and 4.

Surprisingly, our results are almost identical to those calculated by SSQ in most cases. Indeed, although they are fundamentally different, the two methods use the same approximation of the centrifugal term. In most of the other cases, our technique improves on the SSQ results. This is the case for instance, for the $O_{2}\left(X^{3} \Sigma_{g}^{-}\right)$molecule $(n=0,3,5$ and $\ell=10,15)$, for the $O_{2}^{+}\left(X^{2} \Pi_{g}\right)(\ell=10$ and $n=1,3,5 ; \ell=$ $15,20)$, and for the molecules $H_{2}\left(X^{1} \Sigma_{g}^{+}\right)$and $H F\left(X^{1} \Sigma^{+}\right)$. 

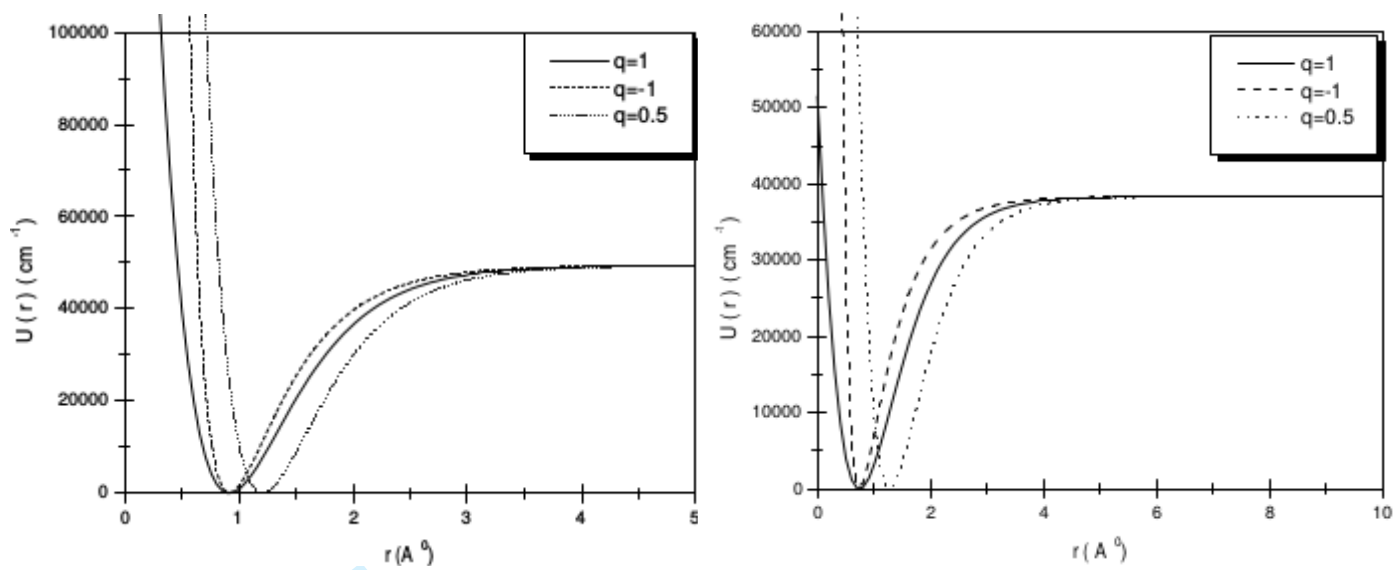

Fig. 1. Variation of the Schiöberg-type potential given in Equation (6) as a function of $r$ for the $H F$ (left) and $H_{2}$ (right) diatomic molecule with three different values of $q$.

\section{Conclusion}

In this work, we presented and improved expression for the $q$-deformed Schiöberg potential energy function using Feynman path integral method and the equivalence between the studied potential and the Tietz-Hua oscillator potential [21]. We applied this method to calculate the spectrum associated to six diatomic molecules $H_{2}\left(X^{1} \Sigma_{g}^{+}\right), H F\left(X^{1} \Sigma^{+}\right), N_{2}\left(X^{1} \Sigma_{g}^{+}\right)$ , $N O\left(X^{2} \Pi_{r}\right), O_{2}\left(X^{3} \Sigma_{g}^{-}\right)$and $O_{2}^{+}\left(X^{2} \Pi_{g}\right)$. Taking the experimental values for the spectroscopic parameters $\left(D_{e}, r_{e}, \eta\right.$ and $\alpha$ ) as inputs, we calculated the numerical values of the energies associated to different $n$ and $\ell$. The obtained eigenvalues are in very good agreement with those given by the supersymmetric and the numerical method which proves the efficiency of our method in solving this type of potentials. In the future, we plan to apply this method to solve the Dirac and the Klein-Gorden equations for more general exponential-type potentials.

\section{References}

1. W., Sun and H., Feng 1999 J. Phys. B : Mol. Opt. Phys. 325109

2. P-M., Morse 1929 Phys. Rev. 3457

3. Z., Rong, H-G., Kjaergaard and M-L., Sage 2003 Mol. Phys. 101 2285

4. J., Welker and F-J., Giessibl 2012 Science. 336444

5. D., Schiöberg 1986 Mol. Phys. 591123

6. S-M., Ikhdair and R., Sever 2009 Ann. Phys. (Berlin) 18189

7. S., Dong, J., Garcia-Ravelo and S-H., Dong 2007 J. Phys. Scr. 76393

8. S., Ortakaya 2013 Few-body Syst. 541901

9. A., Arai 1991 J. Math. Anal. Appl. 15863

10. H., Eğrifes, D., Demirhan and F., Büyükkilic 2000 Phys. Lett. A 275229

11. H., Eğrifes, D., Demirhan and F., Büyükkilic 1999 Phys. Scr. 59 90

12. H., Eğrifes, D., Demirhan and F., Büyükkilic 1999 Phys. Scr. 60 195

13. C-S., Jia, L-Z., Yi and S-W., Long 2014 Can. J. phys. 921258.

14. C-S., Jia, T., Chen, L-Z., Yi and S-R., Lin 2013 J. Math. Chem. 512165

15. H-J., de Vega 1989 Int. J. Mod. Phys. A 42371
16. J., Zhang 2000 J. Phys. Lett. B 477361

17. A., Algin, M., Arik and A-S., Arikan 2002 Phys. Rev. E 65 26140

18. A., Ballesteros, O., Civitarese and M., Reboiro 2003 Phys .Rev. C 6844307

19. Y., Sun, S., He and C-S., Jia 2013 Phys. Scr. 87025301

20. S., Ikhdair, M., Hamzavi and A-A., Rajabi 2013 Int. J, Mod. Phys. E 221350015

21. O., Mustafa 2015 Phys. Scr. 90065002 ; O., Mustafa 2014 arXiv:1409.6986v1

22. Y., Sun, G-D., Zhang and C-S., Jia 2015 Chem. Phys. Lett. 636 197

23. A., Kurniawan, A., Suparmi and A., Cari 2015 Chin. B 24 030302

24. A., Diaf and A., Chouchaoui 2011 Phys. Scr. 84015004

25. A., Diaf 2015 Chin. Phys. B V24, N02 020302

26. A., Diaf and M., Hachama 2013 Can. J. Phys. 911081

27. A-K., Roy 2014 J. Math. Chem. 521405

28. Y-P., Varshni 1957 Rev. Mod. Phys. 29664

29. C-S., Jia, Y-F., Diao, X-J., Liu, P-Q., Wang, J-Y., Liu and G-D., Zhang 2012 J. Chem. Phys. 137, 014101

30. H-M., Tang, G-C., Liang, L-H., Zhang, F., Zhao and C-S., Jia 2014 Can. J. Chem. 92201

31. G-D., Zhang, W., Zhou, J-Y., Liu, L-H., Zhang and C-S., Jia, 2014 Chem. Phys.439 79

32. M., Badawi, N., Bessis and G., Bessis 1972 J. Phys. B: At. Mol. Phys. 5 L157

33. J., Lu 2005 Phys. Scr. V72, 349, 2005

34. P-Q., Wang, J-Y., Liu, L-H., Zhang, S-Y., Cao and C-S., Jia 2012 J. Mol. Spectrosc. 27823 
Table 2. Ro-vibrational energies $E_{n, l}\left(\mathrm{~cm}^{-1}\right)\left(1 \mathrm{~cm}^{-1}=1.239841930 \times 10^{-4} \mathrm{eV}\right)$ for two diatomic molecules $N O\left(X^{2} \Pi_{r}\right)$ and $\mathrm{O}_{2}\left(X^{3} \Sigma_{g}^{-}\right)$with $n=0,3,5$ and different value of $l$.

\begin{tabular}{|c|c|c|c|c|c|c|c|}
\hline \multicolumn{4}{|c|}{$\overline{N O}\left(X^{2} \Pi_{r}\right)$} & \multicolumn{3}{|c|}{$O_{2}\left(X^{3} \Sigma_{g}^{-}\right)$} & \multirow[b]{2}{*}{ Our results } \\
\hline$n$ & $l$ & GPS[27] & SSQ [21] & Our results & GPS[27] & SSQ [21] & \\
\hline \multirow[t]{9}{*}{0} & 0 & 947.759 & 947.756 & 947.757 & 774.984 & 775.089 & 775.090 \\
\hline & 1 & 951.123 & 951.121 & 951.120 & 777.848 & 777.863 & 777.860 \\
\hline & 2 & 957.849 & 957.847 & 957.847 & 783.394 & 783.410 & 783.411 \\
\hline & 3 & & 967.937 & 967.937 & & 791.731 & 791.734 \\
\hline & 4 & & 981.390 & 981.390 & & 802.823 & 802.823 \\
\hline & 5 & & 998.205 & 998.204 & & 816.688 & 816.682 \\
\hline & 10 & 1132.686 & 1132.686 & 1132.686 & 927.562 & 927.578 & 927.574 \\
\hline & 15 & 1351.069 & 1351.072 & 1351.072 & 1107.634 & 1107.654 & 1107.648 \\
\hline & 20 & 1653.146 & 1653.153 & 1653.153 & 1356.714 & 1356.739 & 1356.744 \\
\hline \multirow[t]{9}{*}{3} & 0 & 6453.267 & 6453.239 & 6453.240 & 5269.581 & 5269.672 & 5269.673 \\
\hline & 1 & 6456.510 & 6456.484 & 6456.483 & 5272.250 & 5272.343 & 5272.339 \\
\hline & 2 & 6462.995 & 6462.971 & 6462.972 & 5277.588 & 5277.684 & 5277.684 \\
\hline & 3 & & 6472.703 & 6472.702 & & 5285.694 & 5285.697 \\
\hline & 4 & & 6485.677 & 6485.677 & & 5296.374 & 5296.373 \\
\hline & 5 & & 6501.894 & 6501.894 & & 5309.722 & 5309.715 \\
\hline & 10 & 6631.552 & 6631.592 & 6631.593 & 5416.325 & 5416.479 & 5416.474 \\
\hline & 15 & 6842.080 & 6842.207 & 6842.206 & 5589.607 & 5589.837 & 5589.828 \\
\hline & 20 & 7133.275 & 7133.526 & 7133.525 & 5829.279 & 5829.619 & 5829.623 \\
\hline \multirow[t]{9}{*}{5} & 0 & 9951.736 & 9951.693 & 9951.693 & 8118.378 & 8118.516 & 8118.516 \\
\hline & 1 & 9954.898 & 9954.857 & 9954.858 & 8120.977 & 8121.118 & 8121.113 \\
\hline & 2 & 9961.220 & 9961.188 & 9961.187 & 8126.175 & 8126.321 & 8126.323 \\
\hline & 3 & & 9970.679 & 9970.679 & & 8134.126 & 8134.127 \\
\hline & 4 & & 9983.335 & 9983.335 & & 8144.530 & 8144.529 \\
\hline & 5 & & 9999.155 & 9999.154 & & 8157.535 & 8157.530 \\
\hline & 10 & 10125.542 & 10125.669 & 10125.670 & 8261.257 & 8261.546 & 8261.543 \\
\hline & 15 & 10330.775 & 10331.112 & 10331.112 & 8429.966 & 8430.441 & 8430.433 \\
\hline & 20 & 10614.632 & 10615.269 & 10615.269 & 8663.303 & 8664.046 & 8664.049 \\
\hline
\end{tabular}


Table 3. Ro-vibrational energies $E_{n, l}\left(\mathrm{~cm}^{-1}\right)\left(1 \mathrm{~cm}^{-1}=1.239841930 \times 10^{-4} \mathrm{eV}\right)$ for two diatomic molecules $O_{2}^{+}\left(X^{2} \Pi_{g}\right)$ and $N_{2}\left(X^{1} \Sigma_{g}^{+}\right)$with $n=0,3,5$ and different value of $l$.

\begin{tabular}{cccccccc}
\hline \hline & & $O_{2}^{+}\left(X^{2} \Pi_{g}\right)$ & & & $N_{2}\left(X^{1} \Sigma_{g}^{+}\right)$ \\
\hline$n$ & $l$ & GPS[27] & SSQ[21] & Our results & GPS[27] & SSQ [21] & Our results \\
\hline 0 & 0 & 934.601 & 934.614 & 934.612 & 1174.916 & 1174.927 & 1174.927 \\
& 1 & 937.848 & 937.862 & 937.860 & 1178.870 & 1178.882 & 1178.879 \\
& 2 & 944.341 & 944.353 & 944.354 & 1186.778 & 1186.789 & 1186.788 \\
& 3 & & 954.094 & 954.095 & & 1198.651 & 1198.649 \\
& & & 967.079 & 967.072 & & 1214.466 & 1214.465 \\
& 5 & & 983.310 & 983.306 & & 1234.234 & 1234.240 \\
& 10 & 1113.112 & 1113.127 & 1113.136 & 1392.325 & 1392.338 & 1392.337 \\
& 15 & 1323.924 & 1323.940 & 1323.932 & 1649.087 & 1649.103 & 1649.107 \\
& 20 & 1615.541 & 1615.563 & 1615.544 & 2004.288 & 2004.306 & 2004.305 \\
\hline 3 & 0 & 6376.545 & 6376.615 & 6376.612 & 8047.8758 & 8047.9316 & 8047.9313 \\
& 1 & 6379.684 & 6379.756 & 6379.755 & 8051.7163 & 8051.7736 & 8051.7718 \\
& 2 & 6385.962 & 6386.035 & 6386.034 & 8059.3972 & 8059.4558 & 8059.4546 \\
& 3 & & 6395.455 & 6395.456 & & 8070.9804 & 8070.9783 \\
& 4 & & 6408.015 & 6408.008 & & 8086.3444 & 8086.3428 \\
& 5 & & 6423.713 & 6423.708 & & 8105.5492 & 8105.5556 \\
& 10 & 6549.135 & 6549.270 & 6549.277 & 8259.0350 & 8259.1477 & 8259.1462 \\
& 15 & 6752.948 & 6753.159 & 6753.151 & 8508.4072 & 8508.5904 & 8508.5949 \\
& 20 & 7034.867 & 7035.194 & 7035.186 & 8853.3707 & 8853.6563 & 8853.6550 \\
\hline 5 & 0 & 9845.984 & 9846.089 & 9846.089 & 12460.466 & 12460.549 & 12460.549 \\
& 1 & 9849.051 & 9849.159 & 9849.158 & 12464.229 & 12464.316 & 12464.313 \\
& 2 & 9855.183 & 9855.296 & 9855.294 & 12471.756 & 12471.847 & 12471.845 \\
& & 9864.503 & 9864.502 & & 12483.146 & 12483.143 \\
& & & 9876.778 & 9876.771 & & 12498.208 & 12498.206 \\
& & & 9892.120 & 9892.117 & & 12517.037 & 12517.042 \\
& & & & & & & \\
& 10 & 10014.566 & 10014.830 & 10014.839 & 12667.396 & 12667.620 & 12667.618 \\
& 10213.639 & 10214.091 & 10214.084 & 12911.769 & 12912.164 & 12912.167 \\
& 10488.989 & 10489.719 & 10489.708 & 13249.806 & 13250.446 & 13250.444 \\
\hline \hline
\end{tabular}


Table 4. Ro-vibrational energies $E_{n, l}\left(\mathrm{~cm}^{-1}\right)\left(1 \mathrm{~cm}^{-1}=1.239841930 \times 10^{-4} \mathrm{eV}\right)$ for two diatomic molecules $H_{2}\left(X^{1} \Sigma_{g}^{+}\right)$and $H F\left(X^{1} \Sigma^{+}\right)$with $n=0,3,5$ and different value of $l$.

\begin{tabular}{|c|c|c|c|c|c|c|c|}
\hline \multicolumn{4}{|c|}{$\overline{H_{2}\left(X^{1} \Sigma_{g}^{+}\right)}$} & \multicolumn{3}{|c|}{$\overline{H F F\left(X^{1} \Sigma^{+}\right)}$} & \multirow[b]{2}{*}{ Our results } \\
\hline$n$ & $l$ & GPS[27] & SSQ[21] & Our results & GPS[27] & SSQ [21] & \\
\hline \multirow[t]{9}{*}{0} & 0 & 2171.618 & 2171.661 & 2171.660 & 2047.581 & 2047.549 & 2047.548 \\
\hline & 1 & 2289.372 & 2289.430 & 2289.427 & 2088.368 & 2088.338 & 2088.338 \\
\hline & 2 & 2523.795 & 2523.908 & 2523.907 & 2169.893 & 2169.866 & 2169.865 \\
\hline & 3 & & 2873.012 & 2873.012 & & 2292.033 & 2292.033 \\
\hline & 4 & & 3333.687 & 3333.686 & & 2454.691 & 2454.690 \\
\hline & 5 & & 3902.003 & 3902.002 & & 2657.640 & 2657.640 \\
\hline & 10 & & 8173.796 & 8173.795 & & 4266.461 & 4266.460 \\
\hline & 15 & 14184.547 & 14257.421 & 14257.418 & 6824.964 & 6825.606 & 6825.605 \\
\hline & 20 & 21121.346 & 21406.290 & 21406.287 & 10257.292 & 10259.247 & 10259.246 \\
\hline \multirow[t]{9}{*}{3} & 0 & 13641.123 & 13641.356 & 13641.357 & 13298.712 & 13298.514 & 13298.513 \\
\hline & 1 & 13738.726 & 13740.204 & 13740.202 & 13334.713 & 13334.578 & 13334.577 \\
\hline & 2 & 13932.924 & 13937.042 & 13937.041 & 13406.668 & 13406.661 & 13406.659 \\
\hline & 3 & & 14230.181 & 14230.179 & & 13514.670 & 13514.669 \\
\hline & 4 & & 14617.148 & 14617.144 & & 13658.467 & 13658.465 \\
\hline & 5 & & 15094.757 & 15094.756 & & 13837.868 & 13837.867 \\
\hline & 10 & & 18693.126 & 18693.125 & & 15259.392 & 15259.390 \\
\hline & 15 & & 23846.083 & 23846.079 & & 17518.249 & 17518.247 \\
\hline & 20 & & 29951.340 & 29951.338 & & 20544.186 & 20544.184 \\
\hline \multirow[t]{9}{*}{5} & 0 & 19915.723 & 19916.031 & 19916.031 & 19860.703 & 19860.423 & 19860.421 \\
\hline & 1 & 20000.399 & 20003.596 & 20003.592 & 19893.600 & 19893.479 & 19893.478 \\
\hline & 2 & 20168.807 & 20177.985 & 20177.984 & 19959.350 & 19959.547 & 19959.545 \\
\hline & 3 & & 20437.737 & 20437.735 & & 20058.539 & 20058.538 \\
\hline & 4 & & 20780.713 & 20780.708 & & 20190.325 & 20190.323 \\
\hline & 5 & & 21204.160 & 21204.157 & & 20354.730 & 20354.728 \\
\hline & 10 & & 24399.460 & 24399.458 & & 21656.962 & 21656.960 \\
\hline & 15 & & 28992.500 & 28992.498 & & 23724.509 & 23724.507 \\
\hline & 20 & & 34465.676 & 34465.674 & & 26490.600 & 26490.599 \\
\hline
\end{tabular}

